\title{
Gamma-ray emission from pulsar binaries
}

\author{
John Kirk and Iwona Mochol \\ Max-Planck-Institut für Kernphysik, Germany \\ email: john.kirk@mpi-hd.mpg.de
}

\begin{abstract}
Pulsar winds, containing charged particles, waves and a net (phase-averaged) magnetic field, are thought to fuel the high-energy emission from several gamma-ray binaries. They terminate where the ram pressure matches that of the surroundings - which, in binaries, is provided by the wind of the companion. Before termination, pulsed emission can be produced by inverse Compton scattering of photons from the companion by particles in the waves. After termination, both the bulk kinetic energy of the particles and the Poynting flux in the waves are dissipated into an energetic particle population embedded in the surviving phase-averaged magnetic field. Pulsed emission is no longer possible, but a substantial flux of unpulsed highenergy photons can be produced. I will present results showing that the physical conditions at the termination shock can be divided into two regimes: a high density one, where current sheets in the wind are first compressed by an MHD shock and subsequently dissipate by reconnection, and a low density one, where the wind can first convert into an electromagnetic wave in the shock precursor, which then damps and merges into the wind nebula. The shocks surrounding isolated pulsars fall into the low-density category, but those around pulsars in binary systems, may transit from one regime to the other according to binary phase. The implications of the shock-structure dichotomy for these objects will be discussed.
\end{abstract}

